\title{
Optimum Design of a hybrid PV-CSP-LPG Microgrid with Particle Swarm Optimization Technique
}

\author{
Sara Ghaem Sigarchian ${ }^{\mathrm{a}, 1 *}$, Matthew S. Orosz ${ }^{\mathrm{b}}$, Harry F. Hemond ${ }^{\mathrm{b}}$, Anders Malmquist ${ }^{\mathrm{a}}$ \\ ${ }^{a}$ Department of Energy Technology, KTH Royal Institute of Technology, 10044 Stockholm, Sweden \\ ${ }^{b}$ Parsons Laboratory, Department of Civil and Environmental Engineering, Massachusetts Institute of Technology Cambridge, Massachusetts, 02139, \\ USA
}

\begin{tabular}{|c|c|}
\hline ARTICLE INFO & ABSTRACT \\
\hline Article history: & Designing an energy system using multiple energy sources including renewables and \\
\hline Received & $\begin{array}{l}\text { providing multiple energy services (e.g. electricity, heating) can enhance the reliability } \\
\text { and efficiency of the system while mitigating the environmental footprint. However, }\end{array}$ \\
\hline Received in revised form & interaction among various components, variation of the energy demand profile, and \\
\hline Accepted & $\begin{array}{l}\text { local ambient conditions make design optimization a complex task, and suggesting that } \\
\text { efficient simulation tools and optimization techniques can help designers to determine }\end{array}$ \\
\hline Available online & the best solutions within a reasonable timeframe and budget. \\
\hline Keywords: & $\begin{array}{l}\text { - Previous work on a dynamic microgrid simulation tool called "u-Grid" used an } \\
\text { exhaustive search technique to find optimum configurations. However, the high } \\
\text { computational cost of the exhaustive search was a motivation to explore alternative }\end{array}$ \\
\hline Hybrid energy system & optimization methods to improve the optimization process and also to enhance search \\
\hline Polygeneration & $\begin{array}{l}\text { speed. In this paper Particle Swarm Optimization (PSO) has been presented as a global } \\
\text { optimizer and incorporated within the problem context. Results from the exhaustive }\end{array}$ \\
\hline Optimization & $\begin{array}{l}\text { search have been used as a benchmark for testing and validation of the newly } \\
\text { introduced optimization technique. The result shows that the PSO method is an efficient }\end{array}$ \\
\hline Particle Swarm Optimization & technique which has the ability to determine a high quality design solution for an \\
\hline Microgrid & $\begin{array}{l}\text { optimized microgrid with a relatively low computational cost. Applying this PSO-based } \\
\text { algorithm to the case study has reduced the total computation time a factor of about } 6 \text { in } \\
\text { a significantly smaller computational platform. }\end{array}$ \\
\hline
\end{tabular}

\section{Introduction}

Electricity as a basic service has a significant role in promoting economic development, education, and health of society, and its availability is correlated with increased quality of life. However, 1.5 billion people around the world lack access to electricity; this figure includes many living in isolated areas far from the electric grid.

Providing electricity for these areas in developing countries through a community distributed generation (microgrid) is an increasingly viable solution to address growing energy demand, and may additionally be beneficially deployed where grid reliability is poor or load shedding is common. Implementing a hybrid configuration using multiple energy sources including renewable energy, while providing heat and electricity through cogeneration can increase power availability and efficiency of the system while minimizing its carbon footprint. However, integration of non-dispatchable generators such as solar PV, and variation of the local energy demand profile obviates a "one size fits all" approach. Simulation tools that can accurately assist designers while demanding reasonable amounts of computational resources are needed.

Orosz and Mueller (2015) presented an open source code microgrid performance and component sizing methodology, called "u-Grid," in which an exhaustive search technique is used to find an optimum configuration. This approach has been exercised in a case study optimizing the hybrid configuration of an off-grid energy system for a community in Sub-Saharan Africa [1].

1

Corresponding author. Tel.: +46-8-790-7476;; e-mail: sarags@kth.se

(C) 2016. This manuscript version is made available under the Elsevier user license

http://www.elsevier.com/open-access/userlicense/1.0/ 
However, due the high computational cost of the exhaustive search, in this study we examine other optimization methods as a means to improve the search speed. Amongst them Particle Swarm Optimization (PSO), which is a population based optimization technique, has been identified as a suitable alternative. In this work a PSO-based algorithm is developed and the result of an exhaustive search technique is used as a benchmark for its validation and verification.

System description, simulation methodology and design approach are explained briefly in this paper, and additional details can be found in the previous work [1].

\section{System Description}

The microgrid described in this paper is a hybrid energy system consisting of PV modules, Parabolic, Concentrated Solar Power (CSP), packed bed thermal energy storage (TES), organic Rankine cycle (ORC), electrochemical batteries, and a LPG fueled engine-generator as a backup system with optional exhaust gas waste heat recovery[1].

The power plant topology in a fully integrated hybrid configuration is illustrated in Figure 1.

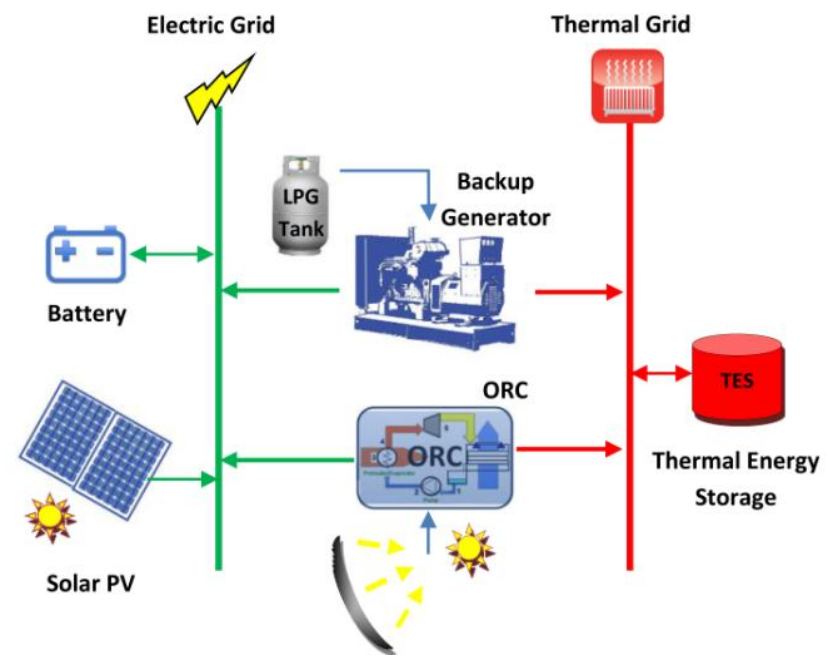

Figure 1 Microgrid schematic showing the range of components considered: LPG Backup Generator, PV Array, Battery

Bank, Concentrating Solar Power (CSP), Thermal Energy Storage (TES) and Organic Rankine Cycle (ORC).

Designing an energy system that integrates several technologies demands a combination of components that can be complementary to each other both technically and economically. For example a PV system has high initial cost, low operation and maintenance cost and low $\mathrm{CO}_{2}$ emission compared to a genset which has the opposite characteristics. Due the intermittent nature of solar energy, a PV system is not dispatchable, and for $100 \%$ availability it must be combined either with storage, a backup generator, or both.

Technically specifying and sizing a combination of different electric power sources is the first step towards design of a viable energy system. However, to make the system economically feasible the optimum configuration of the system should be determined based on a financial figure of merit (e. g. minimized LCOE (Levelized Cost of Energy), maximized IRR (internal rate of return) etc.).

\section{System Simulation}

To simulate the system during one year operation, a coupled energy balance and cash flow model has been implemented in Engineering Equation Solver (EES). The main goal is to determine the best possible configuration including the size of each power source and the optimum operating control strategy that can fulfill the variable energy demand at a minimum cost to users. It is assumed that, barring component failure, no power outage is allowed in the system, meaning that the micro-grid is designed to meet $100 \%$ of load demand.

\section{Objective Function and Parameterization}

The main goal of the design optimization is to identify the optimum power flow control strategy and system infrastructure that achieves a minimum tariff (USD/kWh) to consumers for cost recovery of the capital and operating expenses. Because there are multiple configurations that might have the same tariff value along a Pareto frontier, finding the minimum propane consumption 
has been set as the secondary objective. The user input project finance variables include loan tenure and interest rate, and the local conditions are specified in terms of a load curve and typical meteorological year (TMY) dataset.

Initial capital cost, operation and maintenance cost and replacement cost of each component are included as inbuilt cost functions. In addition, transmission and distribution line, smart metering devices and ICT (Information Communication Technology) solutions for money transaction are also considered in the tariff calculation.

This simplified framework for microgrid design and deployment is based on the assumption of a fee for service (i.e. prepaid smart meter) business model with positive cash flow and returns to debt capital for the power producer (government or private sector), and a level of service at the household connections comparable to the level of service for similar small communities served by the grid.

The optimum configuration with the minimum tariff and fuel consumption can be studied through a sensitivity analysis on e.g., various financial assumptions, installed capacity, etc.

\section{Optimization Techniques}

There are several optimization techniques available that can be used to efficiently determine the preferred solution of a complex problem. Because of their simplicity and relative ease of implementation, population-based algorithms are well suited to solving problems that are characterized by non-linear sets of equations.

Amongst population based methods, genetic algorithm (GA) and particle swarm optimization (PSO) have been well studied for practical applications in the field of energy systems, and several studies have employed them for optimization purposes. A comparative study [2] between GA and PSO using a set of benchmark test problems as well as two space system design optimization problems shows that while GA and PSO both obtain high quality solutions, PSO involves less computational effort and higher efficiency. Another comparative study [3] between PSO and GA applied to an off-grid energy system indicates that PSO outperforms GA considering both the number of iterations as well as CPU utilization time [3]. Based on these findings, PSO was selected for improving the computational efficiency of u-Grid. Because the result from the previous work [1] obtained a solution using exhaustive search, this is used as a benchmark for the PSO algorithm's validation.

\subsection{Exhaustive Search}

An exhaustive search algorithm, also known as brute-force search or generate and test algorithm, tests and evaluates all the possible solutions within a parameter space defined by boundaries and granularity (parameter step size). Implementation is straightforward and will generally identify the solution, if it exists, within the limitations of the parameter space definition. If the parameter space is infinitely granular, the search will converge on the true solution - but this entails a proportionally higher calculation cost. Conversely, if the search space granularity is reduced, exhaustive search converges proportionally faster, but at the expense of less accurate approximation of the true solution. This algorithm is applied for problems with limited size and complexity, or, as in this study, as a baseline to benchmark optimization algorithms needed to reduce computational time.

\subsection{Particle Swarm Optimization}

PSO, introduced by Kennedy and Eberhart [4], is a population-based optimization algorithm invoking natural behavior of particles, inspired by social behavior of bird flocking or fish schooling [5]. This approach has been shown to efficiently solve multidimensional non-linear functions [3].

Each position of an agent or particle in a n-dimensional search space corresponds to a possible solution. PSO algorithm starts with random initial populations within a search space. In order to find the optimum solution, these initial populations are updated in each iteration. The updating process is influenced by the personal experience of each particle as well as its neighbors' experience (personal and global experience, respectively).

The particle current velocity in the search space and its distance from both personal and global best positions are used to update the next move of the particle through the following equations [5]:

$\mathrm{V}(\mathrm{t}+1)=\mathrm{W} . \mathrm{V}(\mathrm{t})+\mathrm{C} 1 * \operatorname{Rand} 1() *(\mathrm{pBest}(\mathrm{t})-\operatorname{Particle}(\mathrm{t}))+\mathrm{C} 2 * \operatorname{Rand} 2() *(\mathrm{gBest}(\mathrm{t})-\operatorname{Particle}(\mathrm{t}))$

Particle $(\mathrm{t}+1)=$ Particle $(\mathrm{t})+\mathrm{V}(\mathrm{t}+1)$

where $\mathrm{t}$ is a pseudo time increment, $\mathrm{V}$ is the velocity and Particle represents the position of the agent. Rand 1 and Rand 2 are two random numbers in the range [ 01 ], and $\mathrm{C} 1$ and $\mathrm{C} 2$ are the acceleration constants that represent the personal and global nature of the swarm. W is inertia weight which was introduced in the modified version of PSO to control the impact of the history of previous velocity on the current velocity. pBest and gBest represent personal and global best respectively.

These parameters have large impact on the calculated performance of optimization system, and therefore have been the focus of prior research. Other optimization algorithms deployed as meta-optimizers [6] can be used to tune the PSO parameters. In a metaoptimizer, the optimization parameters are subject to tuning for each specific problem to maximum search speed. In a 
comprehensive study on identifying optimum parameters for PSO, Pedersen [6] has presented a rubric for selecting parameters for various optimization scenarios; this rubric was the starting point for the current work.

\subsubsection{PSO Parameters Evaluation and Selection}

PSO variables should ideally be tuned for each type of problem (where "type" is recursively defined as the group of problems for which a single set of PSO variables is appropriate) [6]. Determining the optimum PSO parameters for this specific problem has been a major focus of this work and the results obtained can potentially be used as a guide for similar problems. It should be mentioned that increasing the value of inertia weight facilitates searching new areas (exploration) while decreasing W will facilitate local search (exploitation)[7]. Therefore a larger inertia weight at the initial stage and slightly decreasing it in each iteration improves the performance as the search volume collapses.

$\mathrm{C} 1$ and $\mathrm{C} 2$ are acceleration parameters, for which previous research [6] suggests the use of two positive constants, usually equal although some other studies [8] suggest that starting with a small value for $\mathrm{C} 2$ and large value for $\mathrm{C} 1$, then gradually decreasing and increasing them respectively can improve the performance of optimization However, another study indicated that PSOs having constant acceleration coefficients have shorter convergence times and better exploration speeds in comparison with those having time-varying acceleration coefficients [9].

The performance of PSO is also highly influenced by the updated velocity in each iteration. This velocity is generated in a random process; however, its value should be limited to a maximum/minimum value, otherwise the performance of the system will decrease dramatically. Larger maximum velocity at the initial stage of the algorithm helps exploration, and decreasing it gradually amends exploitation.

As a simple rule of thumb, the velocity boundaries (here called Velocity Factor -VF) can be set at a value equal to $10-40 \%$ of the range of the particles, with observation of how it influences the updated particles and feedback. If the problem is unknown, starting with a smaller value can aid in avoid overshooting; however it decreases the speed of convergence. Since this value is problem dependent it requires tuning to find the best trade-off between the speed of convergence and accuracy. The velocity factor can be decreased over time to enhance the local exploitation at the final stage of algorithm.

Velocity Bound=VF*(Particle's upper bound - Particle's lower bound) ()

Shi and Eberhart [10] suggest that a value for $\mathrm{W}$ in the range [0.9 1.2] is advantageous since the probability of failure to find the global optimum is least in this range. Regarding C1 and C2, they also suggested that the integer 2 is a "good" value for both variables. However, since the best value for acceleration constants and inertia weight is problem dependent, the PSO for enhancing $\mathrm{u}$-Grid was tested for different $\mathrm{W}$ in the range [0.8 1.4 ] and acceleration constants in the range [1 4]. $\mathrm{W}$ and $\mathrm{VF}$ are decreased by $5 \%$ in each iteration while $\mathrm{C} 1$ and $\mathrm{C} 2$ remain constant.

According to the experiments described here, the PSO algorithm has the highest performance when $\mathrm{W}=1$ and $\mathrm{C} 1=\mathrm{C} 2$ are in the range [2 3]. Further experiments show that decreasing $\mathrm{C} 1$ and increasing $\mathrm{C} 2$ slightly in each iteration improve the performance of PSO algorithm.

\section{PSO Implementation}

Based on experiment, the values for this problem are assumed to be $\mathrm{C} 1=\mathrm{C} 2=2$ and $\mathrm{W}=1$. The particle convergence towards the global best for different populations for one run with 20 initial populations is shown in Figure 2.

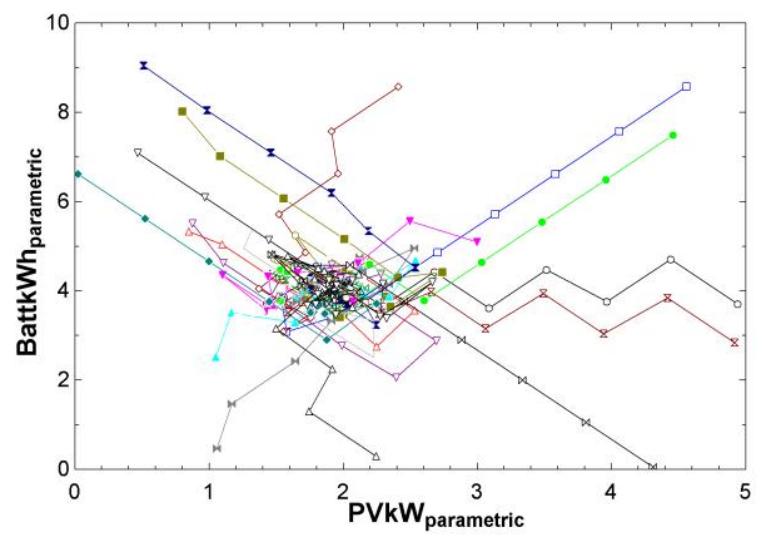


Figure 2 Particles (PV parametric and battery parametric) convergence towards the optimum for a run with 20 populations

Finding the minimum tariff is the primary objective of PSO algorithm and the minimum amount of propane consumption is set to be as the secondary objective. Since a few particles might end up having the same tariff value, the one with the lower amount of propane usage will be set as the global best. The stop criteria of the optimization is having the same tariff for at least three sequential iterations while also attaining parameter values for each particle in the swarm within 5\% of the global best value. The PSO algorithm flowchart is shown in Figure 3.

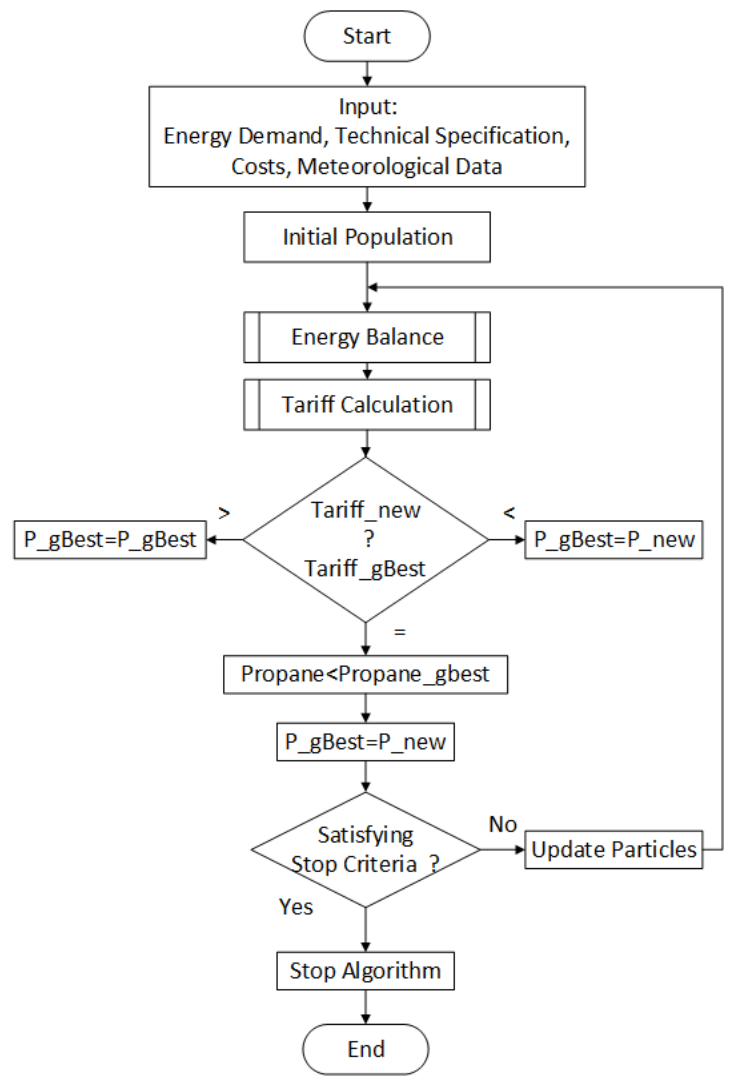

Figure 3 Flow Chart of Particle Swarm Ooptimization algorithm for achieving the minimized cost recovery tariff for a microgrid configuration under positive cash flow scenarios.

7. Simulation result for a case study

This paper revisits the case study community in Lesotho previously investigated in [1]: Ha Nkau, including 84 households, 5 small businesses, a school, a church, and a health clinic. To build a microgrid within the village $5 \mathrm{~km}$ of distribution line with about 18 connections per $\mathrm{km}$ is required [1]. Determining an accurate load demand profile is critical in order to obtain optimized microgrid designs. Based on data available from similar communities, a probabilistic distribution function has been created and processed in an estimation program, detailed in [1]. Using this approach, an annual load profile for the case study has been generated on a 10 minute time step, with an average daily peak load of $45 \mathrm{~kW}$ and average daily consumption of $2.85 \mathrm{kWh}$ day -1 per household (Figure 4

) .

Considering a capital scenario with $5 \%$ return over 15 years, the optimum configuration for the case study community included a PV array of $100 \mathrm{~kW}$ and battery storage of $200 \mathrm{kWh}$ with backup propane consumption of $1636 \mathrm{~kg} / \mathrm{year}$. The minimum tariff is calculated was $0.35 \mathrm{USD} / \mathrm{kWh}$ in order to maintain positive cash flow. These results were obtained through the exhaustive search technique and are used as a benchmark for the PSO optimization algorithm.

A comparative analysis between these two methods demonstrates that using the PSO algorithm has several advantages over exhaustive search technique. In the exhaustive search, all the candidate solutions within the search space must be generated and tested, and the only option for enhanced search speed is parallel computing. For this problem a benchmark computational platform was selected from among Amazon Cloud [11] services: a so-called c4.8xlarge with 60GB of RAM and 36 virtual CPU 
(High frequency Intel Xeon E5-2666 v3 (Haswell) processors ) at the price of $\$ 3.091$ per hour. On this platform 17 program instances were run in parallel and the results are post processed on a standard laptop computer.

In comparison, using the proposed PSO algorithm, defining the search space boundaries is sufficient and there is no need to generate all candidate solutions and subject them to post processing. The PSO was run on a laptop with Intel(R) Core ${ }^{\mathrm{TM}}$ i5-2430M CPU with the speed of $2.5 \mathrm{GHz}$ and the installed memory of $8 \mathrm{~GB}$. The results for comparison of PSO (this work) with exhaustive search are shown in Table 1. It can be seen that a PSO algorithm with a tuned set of parameters is capable of replicating the result of exhausted search at a 6 times faster rate in a significantly smaller computational platform without any hourly payment.

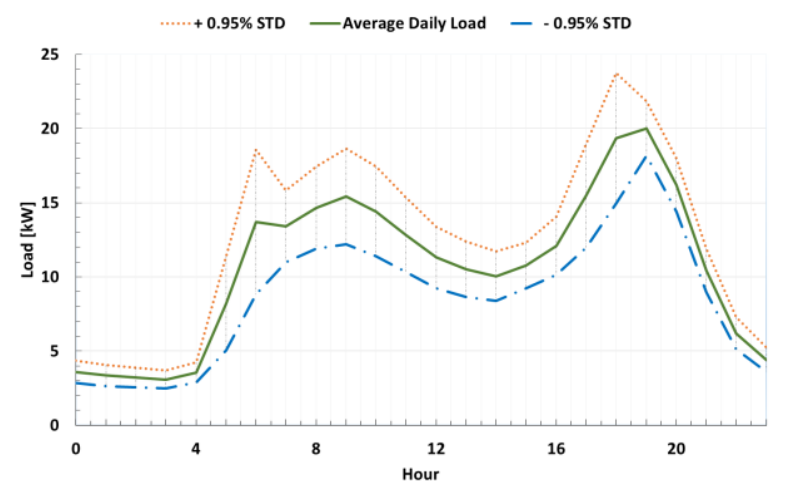

Figure 4 Average inferred daily load curve and standard deviation curves for 84 households and health clinic (Ha Nkau, Lesotho)

Table 1 Result comparison between exhaustive search and PSO

\begin{tabular}{|r|c|c|c|c|c|}
\hline & \multicolumn{4}{|c|}{ Optimal Design } & $\begin{array}{c}\text { Computational } \\
\text { Cost }\end{array}$ \\
\hline Method & $\begin{array}{c}\text { Battery } \\
(\mathrm{KWh})\end{array}$ & $\begin{array}{c}\text { Solar PV } \\
(\mathrm{kW})\end{array}$ & $\begin{array}{c}\text { Tariff } \\
(\mathrm{USD} / \mathrm{kWh})\end{array}$ & $\begin{array}{c}\text { Propane } \\
\text { (kg/year })\end{array}$ & \\
\hline $\begin{array}{c}\text { Exhaustive } \\
\text { Search }\end{array}$ & 200 & 100 & 0.35 & 1636 & 25 Hours \\
\hline PSO & 207 & 99.5 & 0.35 & 1630 & 4 hours \\
\hline
\end{tabular}

\section{Conclusion and future works}

A remote community based hybrid microgrid selecting from among PV, CSP, ORC, sensible TES and battery storage has been simulated using a Particle Swarm Optimization (PSO) method adapted to this study. The resulting design converges on the optimal solution identified by a previous study using exhaustive search with less computational burden (e.g. generating the candidate solutions, running several programs in parallel, sorting results to fine the best solutions). Furthermore the computational speed is enhanced by a factor of 6 using a significantly smaller computational platform without time usage fees. To improve the performance of PSO for this specific problem, PSO parameters were empirically tuned using performance criteria (speed and accuracy).

Future work will include applying this tool for rapid assessment of diverse rural communities in sub-Saharan Africa, India, and other regions where energy access needs are unmet. Future improvement of the design tool will include cogeneration incorporating both electrical and thermal probabilistic distribution functions, and integrating minimum wire cost distribution network design and alternative generation sources (e.g. wind and hydro).

\section{Acknowledgments}

The authors would like to thank KIC InnoEnergy - European Institute of Innovation and Technology to provide financial support through the mobility grant so the author could present at MIT and contribute in this project and also to the STandUp For Energy organization that financially supported the first author.

This (paper) is based upon work supported in part under the US-India Partnership to Advance Clean Energy-Research (PACER) for the Solar Energy Research Institute for India and the United States (SERIIUS), funded jointly by the U.S. Department of Energy (Office of Science, Office of Basic Energy Sciences, and Energy Efficiency and Renewable Energy, Solar Energy Technology Program, under Subcontract DE-AC36-08GO28308 to the National Renewable Energy Laboratory, 
Golden, Colorado) and the Government of India, through the Department of Science and Technology under Subcontract IUSSTF/JCERDC-SERIIUS/2012 dated $22^{\text {nd }}$ Nov. 2012.

\section{References}

[1] M. Orosz and A. V. Mueller, "DYNAMIC SIMULATION OF PERFORMANCE AND COST OF HYBRID PV-CSP-LPG GENERATOR MICRO GRIDS WITH APPLICATIONS TO REMOTE COMMUNITIES IN DEVELOPING COUNTRIES," in Proceeding of the ASME 2015 Power \& Energy Conference, Power Energy 2015, 2015, no. June.

[2] R. Hassan and B. Cohanim, "A comparison of particle swarm optimization and the genetic algorithm," 1st AIAA multidisciplinary design optimization specialist conference, pp. 1-13, 2005.

[3] B. Tudu, S. Majumder, K. K. Mandal, and N. Chakraborty, "Comparative performance study of genetic algorithm and particle swarm optimization applied on off-grid renewable hybrid energy system," Swarm, Evolutionary, and Memetic Computing, vol. 7076, pp. 151-158, 2011.

[4] J. Kennedy and R. Eberhart, "Particle swarm optimization," Neural Networks, 1995. Proceedings., IEEE International Conference on, vol. 4, pp. 1942-1948 vol.4, 1995.

[5] X. Hu, "swarmintelligence.org," Igarss 2014, 2006. [Online]. Available: http://www.swarmintelligence.org.

[6] M. Erik, H. Pedersen, and M. E. H. Pedersen, "Good parameters for particle swarm optimization," Technical Report HL1001, Hvass Laboratories, vol. HL1001, pp. 1-12, 2010.

[7] Y. Shi and R. C. Eberhart, "Parameter Selection in Particle Swarm Optimization," Evolutionary Programming VII SE - 57, vol. 1447, pp. 591-600, 1998.

[8] P. K. Tripathi, S. Bandyopadhyay, and S. K. Pal, "Multi-Objective Particle Swarm Optimization with time variant inertia and acceleration coefficients," Information Sciences, vol. 177, no. 22, pp. 5033-5049, Nov. 2007.

[9] A. Sengupta and V. K. Mishra, "Time Varying vs. Fixed Acceleration Coefficient PSO Driven Exploration during High Level Synthesis: Performance and Quality Assessment," 2014 International Conference on Information Technology, pp. 281-286, 2014.

[10] Y. Shi and R. Eberhart, "A modified particle swarm optimizer," 1998 IEEE International Conference on Evolutionary Computation Proceedings. IEEE World Congress on Computational Intelligence (Cat. No.98TH8360), pp. 69-73, 1998.

[11] “Amazon Web Services.” [Online]. Available: https://aws.amazon.com/console/. 